\title{
Key material parameters driving CBRAM device performances
}

\author{
Ludovic Goux, ${ }^{\text {*a }}$ Janaki Radhakrishnan, ${ }^{a}$ Attilio Belmonte, ${ }^{a}$ \\ Thomas Witters, ${ }^{a}$ Wouter Devulder, ${ }^{a}$ Augusto Redolfi, ${ }^{a}$ Shreya Kundu, ${ }^{a}$ \\ Michel Houssa ${ }^{b}$ and Gouri Sankar Kar ${ }^{a}$
}

Received 5th June 2018, Accepted 19th June 2018

DOI: $10.1039 / c 8 f d 00115 d$

This study is focused on Conductive Bridging Random Access Memory (CBRAM) devices based on chalcogenide electrolyte and Cu-supply materials, and aims at identifying the key material parameters controlling memory properties. The CBRAM devices investigated are integrated on CMOS select transistors, and are constituted by either $\mathrm{Ge}-\mathrm{Se}$ or $\mathrm{Ge}-\mathrm{Te}$ electrolyte layers of various compositions combined with a $\mathrm{Cu}_{2} \mathrm{GeTe}_{3}$ active chalcogenide electrode. By means of extensive physical and electrical characterization, we show for a given electrolyte system that slower write is obtained for a denser electrolyte layer, which is directly correlated with a lower atomic percentage of the chalcogen element in the layer. We also evidence that the use of $\mathrm{Ge}-\mathrm{Se}$ electrolyte results in larger write energy (voltage and time), however with improved state retention properties than for $\mathrm{Ge}-\mathrm{Te}$ electrolyte materials. We associate these results with the stronger chemical bonding of $\mathrm{Cu}$ with $\mathrm{Se}$, resulting both in a stabilized $\mathrm{Cu}$ filament and a slower $\mathrm{Cu}$ cation motion. More robust processing thermal stability is also observed for Ge-Se compared to Ge-Te compounds, allowing more flexibility in the integration flow design.

\section{Introduction}

Conductive Bridging Random Access Memory (CBRAM) is an attractive candidate for storage class and embedded memory applications due to the simple metalinsulator-metal (MIM) structure, scalability, large endurance and fast switching..$^{1-4}$ It operates by the voltage-controlled electrochemical formation (set) and dissolution (reset) of a metallic conductive filament (CF) in a solid electrolyte. After set switching, the CF formation results in a low resistance state (LRS), while after reset switching, the ruptured CF results in a high resistance state (HRS).

The typical CBRAM stack consists of three elements: (1) an active electrode; (2) a solid electrolyte; and (3) an inert electrode. The active electrode supplies the mobile cations $\left(\right.$ e.g. $\left.\mathrm{Cu}^{+}, \mathrm{Ag}^{+}\right)$that are driven by the electric field through the 
electrolyte towards the inert electrode, which should not be electrochemically active in the $\mathrm{CF}$ formation mechanism (e.g. TiN, Pt, W). The mechanism sequence of oxidation, drift, reduction and nucleation in the CF formation is a complex process which depends on the cation mobility and reduction rate in a given electrolyte material. Depending on the materials used, nucleation and CF growth may be initiated already in the electrolyte bulk or only at the inert-electrode interface.

The solid electrolyte is typically an ion-conducting oxide or chalcogenide. The electrolyte is a key element in the CBRAM stack, as it will primarily impact the cation mobility as well as the CF stability after state programming.

Amorphous chalcogenides are attractive electrolyte candidates because they generally allow a large memory window to be obtained $\left(>10^{4}\right)$ using low switching voltages $(<0.5 \mathrm{~V}) .{ }^{5}$ They allow large cation mobility and dissolution, which results in an extensive erasure of the CF during reset and therefore a large HRS resistance level. However, reports on chalcogenide-based CBRAM devices generally show operating currents larger than $100 \mu \mathrm{A},{ }^{6}$ and typically exhibit long write times $(>10$ $\mu \mathrm{s}) .{ }^{6,7}$ Finally, many chalcogenide materials have a low crystallization temperature, and thus their thermal stability needs to be looked after in view of device integration within back-end-of-line processes, which generally require temperatures as high as $400{ }^{\circ} \mathrm{C}$.

In the literature, most reported chalcogenide materials constituting CBRAM electrolytes are Ge-Te, ${ }^{8}$ Ge-Se, ${ }^{1,5,6}$ and Ge-S ${ }^{3,7}$ compounds. Regarding Ge-Te, reproducible and high resistance-window switching has been reported for $\mathrm{Cu} /$ GeTe cells, ${ }^{8}$ however to our knowledge no extensive reliability was reported on this material. The Ge-Se family was also widely reported, showing a high memory window and long retention at low operating current, however using a Ag-supply layer. ${ }^{1,5} \mathrm{Cu} / \mathrm{GeSe}$ stacks were also reported, ${ }^{6}$ however using high operating currents of $200 \mu \mathrm{A}$.

Finally, despite the lower mobility of $\mathrm{Cu}$ cations with respect to $\mathrm{Ag}$ cations in chalcogenide glasses ${ }^{9}$ as well as the more promising properties obtained using Ag-supply, it is desirable to understand and develop Cu-based CBRAM for better CMOS compatibility and integration aspects.

To summarize, a lot of studies have been carried out, however a complete study comparing the performances of Ge-Se and Ge-Te in the same device structure and fabrication conditions is still missing, and would be required to understand the material parameters controlling the CBRAM device and reliability properties. Moreover, it is desirable that this investigation is carried out using a Cu-supply element for increased industrial relevance.

In this study we evaluate the electrical performances of Cu-based CBRAM devices based on chalcogenide electrolyte and supply materials. We compare the device properties using either Ge-Te or Ge-Se chalcogenide layers, aiming at understanding the impacts of the chalcogen content and the chalcogen element. This study has been made using $\mathrm{Cu}_{2} \mathrm{GeTe}_{3}$ (CGT) Cu-supply single-phase compound, which was demonstrated to exhibit good thermal stability and improved $\mathrm{CF}$ retention properties over $\mathrm{Cu}$-Te compounds, using an $\mathrm{Al}_{2} \mathrm{O}_{3}$ electrolyte. ${ }^{10}$ We use this chalcogenide alloy for the first time combined with chalcogenide electrolytes, and we also benchmark the electrical results obtained with pure $\mathrm{Cu}$-based counterparts. 
Based on the material characterization of the different chalcogenide layers, we optimize the deposition process and also derive an integration flow ensuring integrity of the material stacks, allowing guidelines for device improvements to be suggested.

The paper is structured as follows: in the first section, we describe the chalcogenide layer processing optimization and physical characterization, together with preliminary electron-beam structured cell evaluation. In the second section an appropriate integration flow is designed to fabricate scaled devices, and electrical forming and switching DC characterization is studied for all stacks. Finally, the third section is dedicated to electrical pulse switching, write speed and retention properties.

\section{Process and material optimization}

\section{Layer characteristics}

The Ge-Te, Ge-Se, and CGT layers were deposited by a co-sputtering process at room temperature (RT) from $\mathrm{Ge}, \mathrm{Te}, \mathrm{GeSe}_{2}$, and $\mathrm{Cu}_{2} \mathrm{Ge}$ targets. Due to the low thermal stability of Te-rich Ge-Te compounds, we focused our study on the Gerich composition range. For appropriate material comparison we also studied the Ge-rich region for Ge-Se layers.

The layers were deposited in a thickness range between 20 and $50 \mathrm{~nm}$. From Xray reflectometry (XRR), for all compositions studied the thickness was uniform on the wafer, with a standard deviation $<2 \mathrm{~nm}$, and low roughness of less than $1 \mathrm{~nm}$ was measured by atomic force microscopy (AFM). The composition was characterized by electron recoil detection (ERD), Rutherford backscattering (RBS) and proton-induced X-ray emission (PIXE). The developed process recipes resulted in the following composition options: $\mathrm{Ge}_{50} \mathrm{Te}_{50}, \mathrm{Ge}_{65} \mathrm{Te}_{35}$, and $\mathrm{Ge}_{80} \mathrm{Te}_{20}$ for the Ge-rich Ge-Te family, and $\mathrm{Ge}_{60} \mathrm{Se}_{40}$ and $\mathrm{Ge}_{70} \mathrm{Se}_{30}$ for the Ge-rich Ge-Se family.

\section{Preliminary electron-beam cell evaluation}

In order to assess the potential of Ge-Te layers for CBRAM application, we carried out a preliminary evaluation by structuring small GeTelCu cells by an electronbeam (e-beam) lithography technique on CMOS select transistors. The substrate consisted of the front-end process transistor contacted by $\mathrm{W}$ plugs to TiN metal lines planarized by a chemical-mechanical planarization (CMP) technique (Fig. 1a). Subsequently, a resist-coating and e-beam lithography process was carried out to pattern a cross-bar structure. Then a $30 \mathrm{~nm}$-thick $\mathrm{Ge}_{50} \mathrm{Te}_{50}$ layer was deposited by sputtering followed by a $10 \mathrm{~nm} \mathrm{Cu}$ layer capped with $20 \mathrm{~nm}$ TiN, also deposited by sputtering at RT. Finally, the cross-bar structure (Fig. 1a) was obtained by resist lift-off. For comparison, some cells were also fabricated without a $\mathrm{Cu}$ layer.

While a forming voltage $\left(V_{\mathrm{f}}\right)$ of $9 \mathrm{~V}$ was required for $55 \mathrm{~nm}$-size cells without $\mathrm{Cu}, V_{\mathrm{f}}<3 \mathrm{~V}$ was required for cells with a $\mathrm{Cu}$ top electrode (TE) (Fig. 1b). In addition, no bipolar switching was obtained for the former, while the latter cells exhibited controlled DC current-voltage $(I-V)$ switching characteristics at $50 \mu \mathrm{A}$ compliance, as shown in Fig. 1c. This result confirms the CBRAM operation of the stack, requiring $\mathrm{Cu}$ injection at forming for electrical-induced set and reset operations on a $\mathrm{Cu}$-based $\mathrm{CF}$ within the GeTe electrolyte layer. 

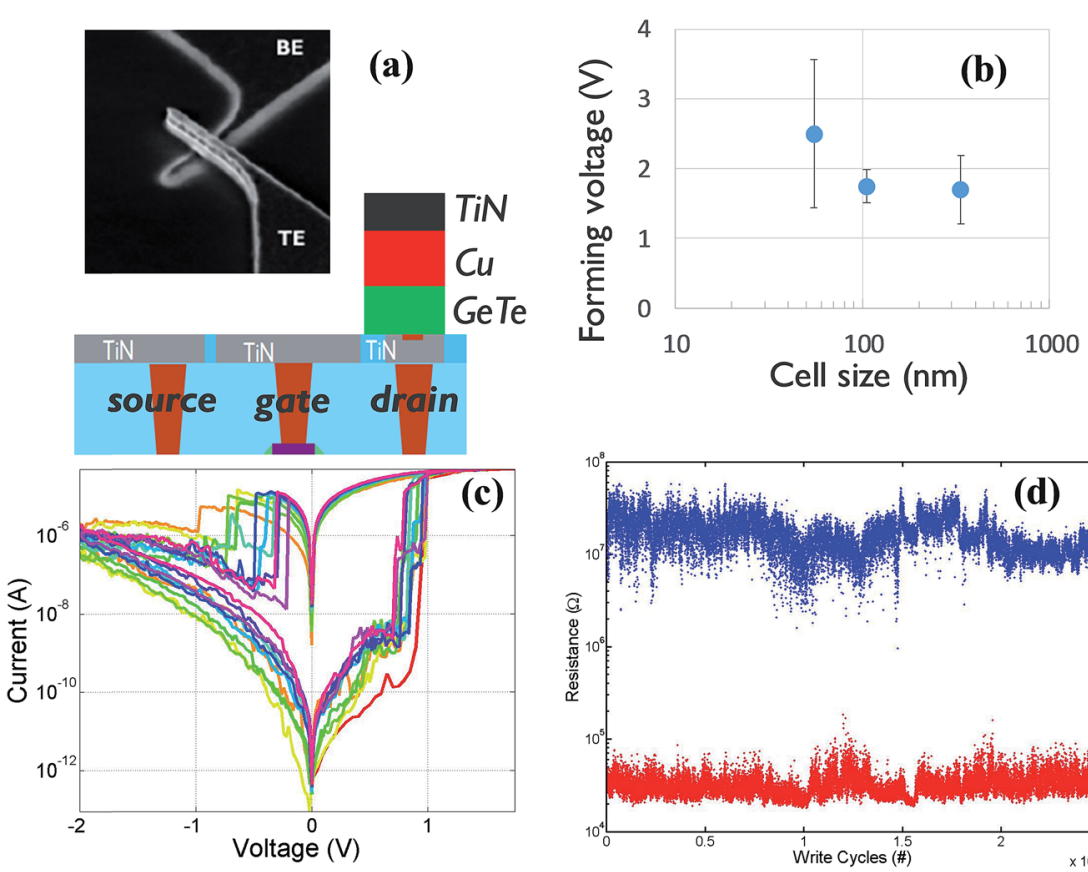

Fig. 1 (a) Schematic of the e-beam processed cross-bar GeTelCu cells; (b) cell-size dependence of the forming voltage obtained by voltage ramp; (c) typical DC I-V switching curves for a transistor-controlled compliance current of $50 \mu \mathrm{A}$; (d) typical AC pulseswitching characteristics obtained using $1 \mu$ s-long set/reset pulses limited to $50 \mu \mathrm{A}$.

We also performed pulse-switching measurements on $55 \mathrm{~nm}$-size GeTe $\backslash \mathrm{Cu}$ cells using a Keithley pulsed $I-V$ instrument. Fig. $1 \mathrm{~d}$ shows typical endurance characteristics obtained using $2.7 \mathrm{~V}$ set and $-2 \mathrm{~V}$ reset amplitudes with pulse width $\mathrm{PW}=1 \mu \mathrm{s}$. Clearly, good switching control and stability is obtained beyond $10^{4} \mathrm{set} /$ reset cycles. Moreover, this result confirms that a large HRS/ LRS resistance window RW $>10^{2}$ may be obtained using chalcogenide electrolytes.

\section{Thermal stability}

Ge-Te chalcogenide compounds, however, have a rather low glass transition temperature and therefore their thermal stability requires careful attention during back-end-of-line (BEOL) processing. For example, the crystallization temperatures for GeTe and $\mathrm{Ge}_{2} \mathrm{Se}_{3}$ were reported as being $182{ }^{\circ} \mathrm{C}$ and $327{ }^{\circ} \mathrm{C}$, respectively. ${ }^{11,12}$ In this regard, we optimized the layers in view of a BEOL thermal budget lower than $250{ }^{\circ} \mathrm{C}$, which was developed concomitantly.

Layers of both Ge-Te and Ge-Se families were deposited on $\mathrm{SiO}_{2}$ substrates (Fig. 2a), and then annealed at $250{ }^{\circ} \mathrm{C}$ for $5 \mathrm{~min}$ in a $\mathrm{N}_{2}$ atmosphere. Fig. $2 \mathrm{~b}-\mathrm{f}$ show grazing-incidence X-ray diffraction (GI-XRD) diagrams obtained for all compositions after annealing. Between all samples, only $\mathrm{Ge}_{50} \mathrm{Te}_{50}$ layers showed crystallization peaks after annealing, related to the formation of the rhombohedral phase of GeTe. 
(a)
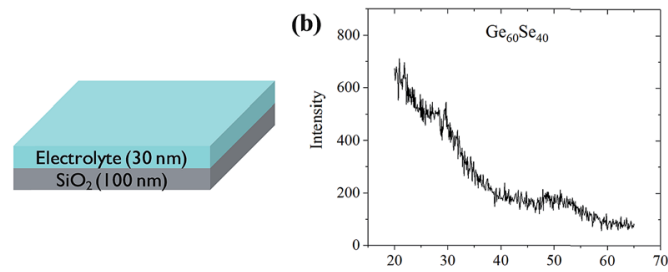

(d)

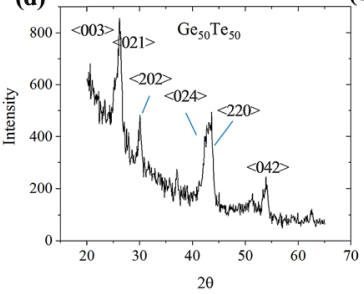

(e)

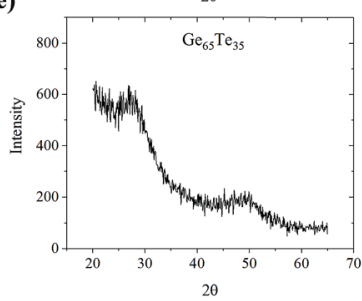

Fig. 2 (a) Schematic of the samples prepared for annealing and GI-XRD characterization; (b-f) GI-XRD diagrams obtained after annealing at $250{ }^{\circ} \mathrm{C}$ for all investigated compositions.

Nitrogen doping has been demonstrated to increase the crystallization temperature of GeTe. ${ }^{13}$ The presence of nitrogen in the amorphous network increases the activation barrier for the crystallization process. It is hypothesized that the higher activation barrier arises from the additional energy required to separate the Ge-N and Ge-Te phases. In addition, the Ge-N may also pile up at the grain boundaries to hinder crystallization. ${ }^{13}$

Based on this, we developed a co-sputtering process in $\mathrm{N}_{2}$ atmosphere using various flow rates (from 5 to $50 \mathrm{sccm}$ ). The atomic percentage of nitrogen in these layers was extracted from ERD analysis. In situ XRD (IS-XRD) performed during temperature ramp-up was also carried out to extract the crystallization temperature for different doping levels. Fig. 3a shows for $\mathrm{Ge}_{50} \mathrm{Te}_{50}(\mathrm{~N})$ layers that the diffraction peaks of the rhombohedral phase appear only above $280{ }^{\circ} \mathrm{C}$ for a doping level of 5.7 at $\% \mathrm{~N}$ (obtained for $20 \mathrm{sccm}$ nitrogen flow). This beneficial

(a) Variation of XRD intensities in function of temperature

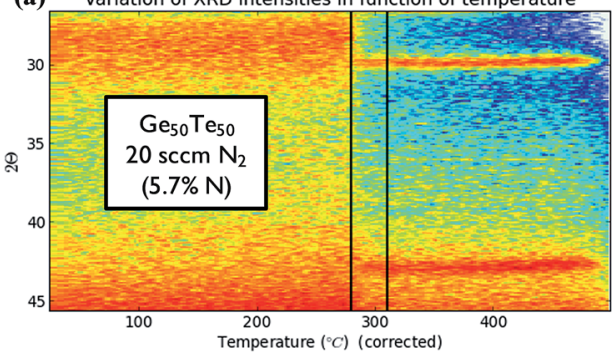

(b)

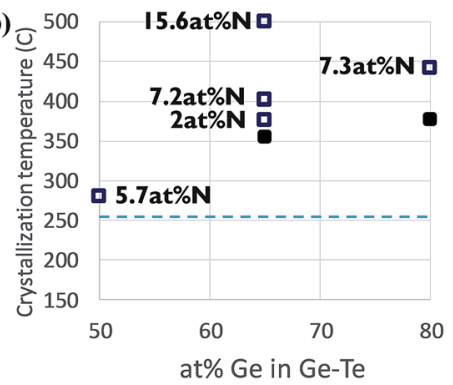

Fig. 3 (a) IS-XRD diagram of 5.7 at\% N-doped GeTe layers, showing successful increase of the crystallization temperature above $280{ }^{\circ} \mathrm{C}$; (b) summary plot of crystallization temperatures obtained for $\mathrm{N}$-doped $\mathrm{Ge}$-Te layers of various compositions and doping levels (closed symbols represent un-doped layers). 
increase of the crystallization temperature is also observed for Ge-richer compositions, as summarized in Fig. $3 \mathrm{~b}$.

Hence, nitrogen doping proves effective in view of our integration flow, and will be used only for the $\mathrm{Ge}_{50} \mathrm{Te}_{50}$ composition hereafter, to a doping level of 5.7 at $\%$.

Note finally that we observed no significant impact of the doping process on the layer characteristics, namely thickness, roughness and composition.

\section{Device integration and electrical characterization methodology}

Using the same CMOS front-end process as for e-beam structured cells, we modified the subsequent flow as follows: instead of fabricating TiN metal lines stacked on the $\mathrm{W}$ plugs (Fig. 1a), we processed a conventional $\mathrm{W}$ damascene module, on top of which we developed a TiN pillar module wherein the physicalvapor-deposited (PVD) TiN layer is patterned, covered with $\mathrm{SiO}_{2}$ and planarized using CMP. Then the CBRAM stack is deposited by in situ sputtering without vacuum break between layers. It consists of the electrolyte layer deposition, followed by a $3 \mathrm{~nm}$-thin Ta layer used to buffer the $\mathrm{Cu}$ in-diffusion, ${ }^{\mathbf{4 , 1 4}}$ and then by the CGT deposition capped with a Ta|Ru।TiN metal stack used as hard-mask for ion beam etch (IBE) patterning of the CBRAM device. Finally the low-temperature $\left(250{ }^{\circ} \mathrm{C}\right)$ passivation and bond-pad processing modules are fabricated.

Fig. $4 \mathrm{a}$ and $\mathrm{b}$ show the electrical schematic of the 1-transistor/1-resistor (1T1R) device configuration and the CBRAM 'mushroom'-shaped stack respectively, while Fig. 4c lists the different electrolyte composition and thickness combinations explored. Note that only the $\mathrm{Ge}_{50} \mathrm{Te}_{50}$ composition is doped with nitrogen, and that it will still be labelled as $\mathrm{Ge}_{50} \mathrm{Te}_{50}$ hereafter.

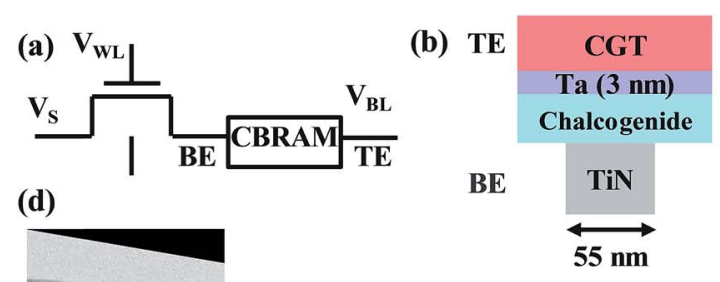

(c) \begin{tabular}{|c|c|}
\hline Electrolyte & Thickness \\
\hline $\begin{array}{c}\mathrm{Ge}_{50} \mathrm{Te}_{50} \\
\left(20 \mathrm{sccm} \mathrm{N}_{2}\right)\end{array}$ & $40 \mathrm{~nm}$ \\
\hline $\mathrm{Ge}_{65} \mathrm{Te}_{35}$ & $40 \mathrm{~nm}$ \\
\hline $\mathrm{Ge}_{80} \mathrm{Te}_{20}$ & $40 \mathrm{~nm}$ \\
\hline $\mathrm{Ge}_{60} \mathrm{Se}_{40}$ & $40,20 \mathrm{~nm}$ \\
\hline $\mathrm{Ge}_{70} \mathrm{Se}_{30}$ & $40,20 \mathrm{~nm}$ \\
\hline
\end{tabular}
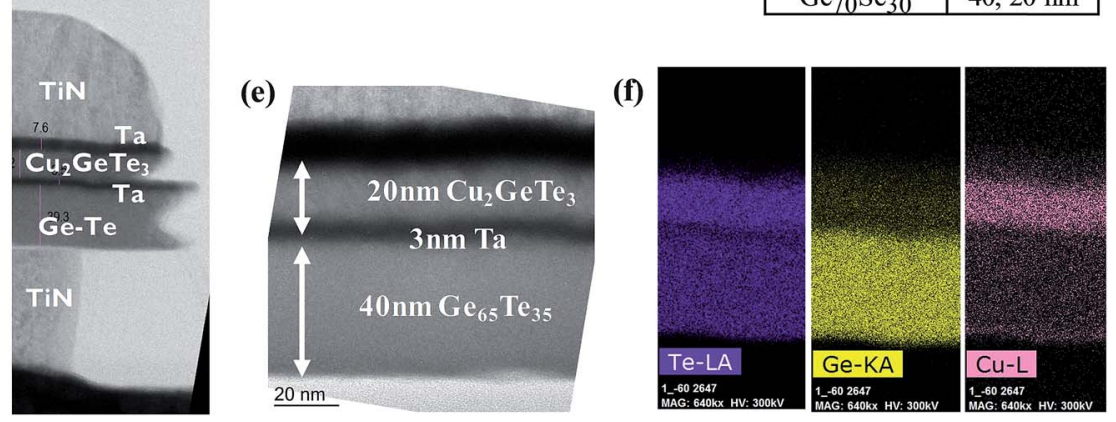

Fig. 4 (a) Electrical schematic of the 1T1R CBRAM cell configuration; (b) material stack constituting the CBRAM 'mushroom'-shaped device; (c) various electrolyte compositions and thicknesses explored; (d) TEM cross-section of a CBRAM device; (e) focused TEM image of the active CBRAM layers; (f) 2-dimensional elemental mappings obtained by EDS during TEM characterization. 
The electrically active area of the mushroom device is determined by the TiN pillar size, having variations down to $55 \mathrm{~nm}$. The IBE patterning steps generate non-uniform device sidewalls, as shown on the transmission-electron microscopy (TEM) image in Fig. 4d, however remotely from the active area and therefore with no impact on the CBRAM properties. Fig. 4e shows the very CBRAM stack in this active area, confirming integrity of the layers and clean interfaces between the layers. The TEM characterization confirmed that all Ge-Te electrolyte compositions show amorphous microstructure after integration, while the CGT layer is crystalline, as expected for a thermal budget of $250{ }^{\circ} \mathrm{C} .{ }^{10}$

Finally, Fig. 4f shows the elemental mapping obtained by electron dispersion spectroscopy (EDS) analysis carried out during TEM imaging of a $\mathrm{Ge}_{65}$ $\mathrm{Te}_{35} \backslash \mathrm{Ta} \backslash$ CGT device. It can be observed that $\mathrm{Cu}$ has clearly diffused into the Ge-Te layer. From the EDS analysis, $\mathrm{Cu}$ amounts to a level of $\sim 5$ at $\%$ in the GeTe layer, which will have some electrical impact as will be shown in the next section.

The electrical characterization was carried out on $1 \mathrm{~T} 1 \mathrm{R}$ cells, where the CBRAM device is $55 \mathrm{~nm}$ in size. DC electrical characterization was carried out using an Agilent B1500A parameter analyzer. Pulse characterization was performed using a dedicated setup consisting of an Agilent 81110A pulse generator and a Keithley 2602A DC source meter. The devices were operated at a maximum current of $50 \mu \mathrm{A}$, unless otherwise stated, controlled by the selecttransistor gate voltage, referred to as word line (WL) in Fig. 4a. The voltage ramps or pulses were applied on the TE terminal of the cell (bit-line) while the source was grounded $\left(V_{\mathrm{S}}=0\right)$. Both the applied voltage pulses and the switching current were monitored using an oscilloscope. After set/reset write sequences, the states were read at $V_{\text {read }}=0.1 \mathrm{~V}$ applied on the TE and using the sourcemeter instrument.

\section{Forming and DC switching characterization}

\section{0 nm-thick chalcogenide layers}

The forming and DC electrical switching behaviors of integrated 1T1R devices are investigated in this section. The memory element consists of $40 \mathrm{~nm}$-thick chalcogenide electrolyte and a $20 \mathrm{~nm}$-thick $\mathrm{Cu}_{2} \mathrm{GeTe}_{3}$ active electrode. Positive voltage ramps were applied on the pristine devices using a ramp rate of $1 \mathrm{~V} \mathrm{~s}^{-1}$, and the forming voltage $V_{\mathrm{f}}$ was extracted.

Fig. 5 a shows the forming voltage cumulative distribution functions (CDF) for each stack. The low spread of the $V_{\mathrm{f}}$ distributions indicates low device-to-device variability in the pristine state, which corresponds to good process control. The median forming curves of the Ge-Te and Ge-Se compositions are plotted in Fig. $5 \mathrm{~b}$ and c respectively.

$V_{\mathrm{f}}$ increases with decreasing chalcogen content in Ge-Te. In contrast, $V_{\mathrm{f}}$ decreases with decreasing chalcogen content in Ge-Se. Note that for $\mathrm{Ge}_{50} \mathrm{Te}_{50} V_{\mathrm{f}}$ is significantly decreased compared to e-beam structured counterpart cells, which is related to the substantial $\mathrm{Cu}$ in-diffusion evidenced for integrated devices (Fig. 4f).

After forming, the devices are reset and set by applying negative (from 0 to -2 $\mathrm{V}$ ) and positive (from 0 to $3 \mathrm{~V}$ ) voltage ramps to the TE respectively. The reset voltage is limited to $-1 \mathrm{~V}$ for $\mathrm{Ge}_{50} \mathrm{Te}_{50}$ devices due to its low breakdown voltage 
(a)
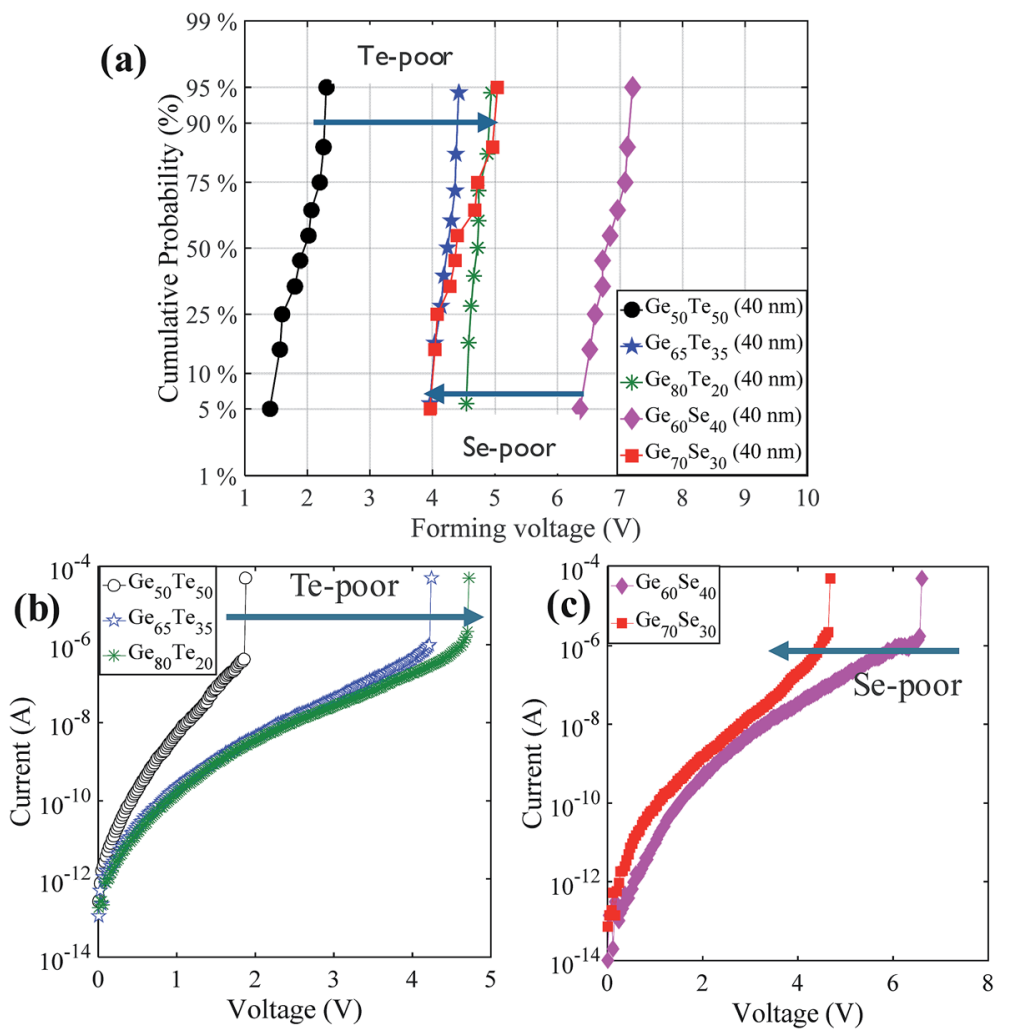

Fig. 5 (a) Cumulative $V_{f}$ distribution plots showing the composition impact for $\mathrm{Ge}-\mathrm{Te}$ and $\mathrm{Ge}$-Se systems; (b and c) median I-V forming curves obtained for $40 \mathrm{~nm}$-thick Ge-Te (b) and $\mathrm{Ge}-\mathrm{Se}$ (c) devices.

$(\sim-1.2 \mathrm{~V})$. Fig. 6 shows the median current-voltage $(I-V)$ switching characteristics. For both Ge-Te and Ge-Se material systems, the set voltage increases with decreasing chalcogen content.
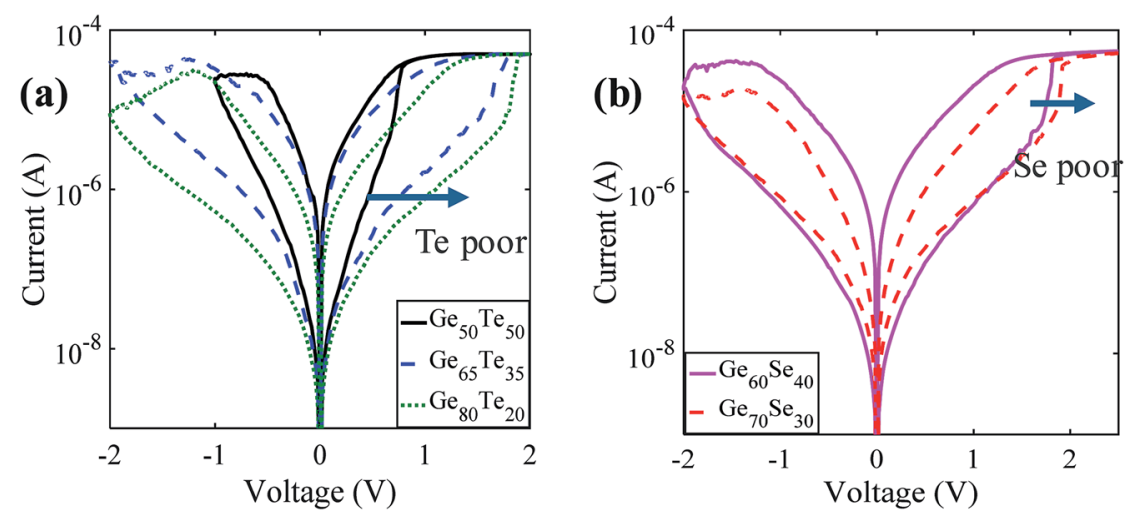

Fig. 6 Median I-V switching curves (10 devices, 10 cycles) obtained for different compositions of $40 \mathrm{~nm}$-thick (a) Ge-Te and (b) Ge-Se layers. 
Thickness impact for Ge-Se layers

The $20 \mathrm{~nm}$-thick Ge-Se layers were investigated in order to reduce $V_{\mathrm{f}}$ below $5 \mathrm{~V}$. The median forming curves for $40 \mathrm{~nm}$ - and $20 \mathrm{~nm}$-thick Ge-Se devices are shown in Fig. 7a. As expected, $V_{\mathrm{f}}$ is decreased by $\sim$ half for $20 \mathrm{~nm}$ layers, suggesting a field-driven forming process.

Fig. $7 \mathrm{~b}$ shows the thickness impact on median switching curves subsequent to forming for $\mathrm{Ge}_{60} \mathrm{Se}_{40}$, while Fig. 7c and d show the impact of the composition on the state distributions for both 40 and $20 \mathrm{~nm}$ thicknesses. Clearly, the memory window is increased by $>10^{3}$ by reducing from 40 to $20 \mathrm{~nm}$ the Ge-Se layer thickness. As this effect is observed primarily on the HRS distribution, it may be attributed to more efficient reset operation.

Note also that the switching variability is larger for a thinner electrolyte layer (Fig. 7d).

\section{CBRAM operation vs. intrinsic switching of the chalcogenide layers}

Similarly to e-beam structured cells, some devices were also integrated without a Cu-supply element in order to decouple potential intrinsic switching effects from effective CBRAM operation.
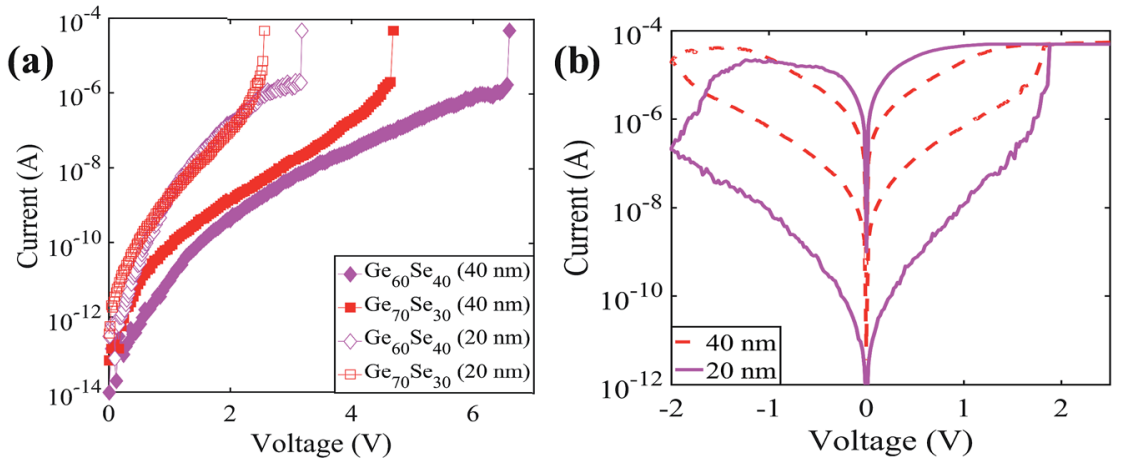

(c)

(d)
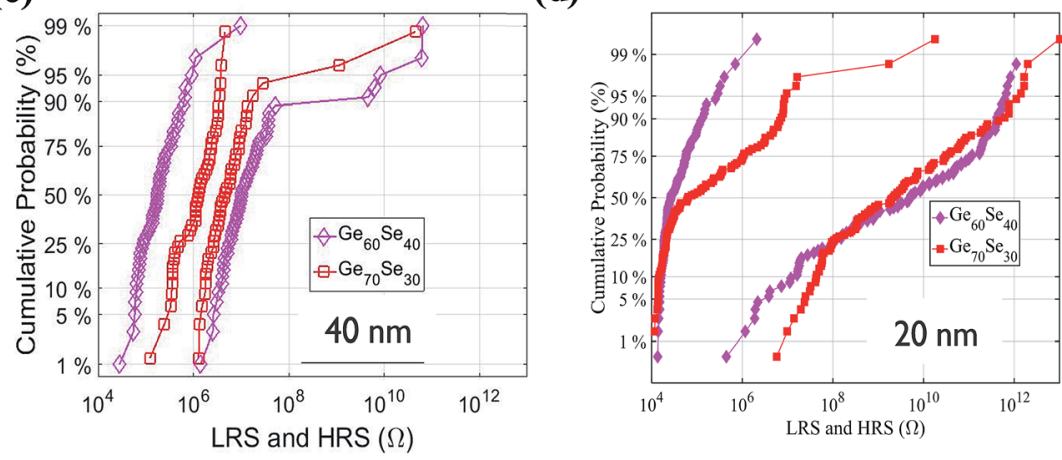

Fig. 7 (a) Median forming curves for $20 \mathrm{~nm}$ - and $40 \mathrm{~nm}$-thick Ge-Se layers; (b) median switching curves for $20 \mathrm{~nm}$ - and $40 \mathrm{~nm}$-thick $\mathrm{Ge}_{60} \mathrm{Se}_{40}$ layers (10 devices, 10 cycles); (c and d) LRS and HRS resistance distribution for $40 \mathrm{~nm}$ - and $20 \mathrm{~nm}$-thick Ge-Se layers, respectively. 

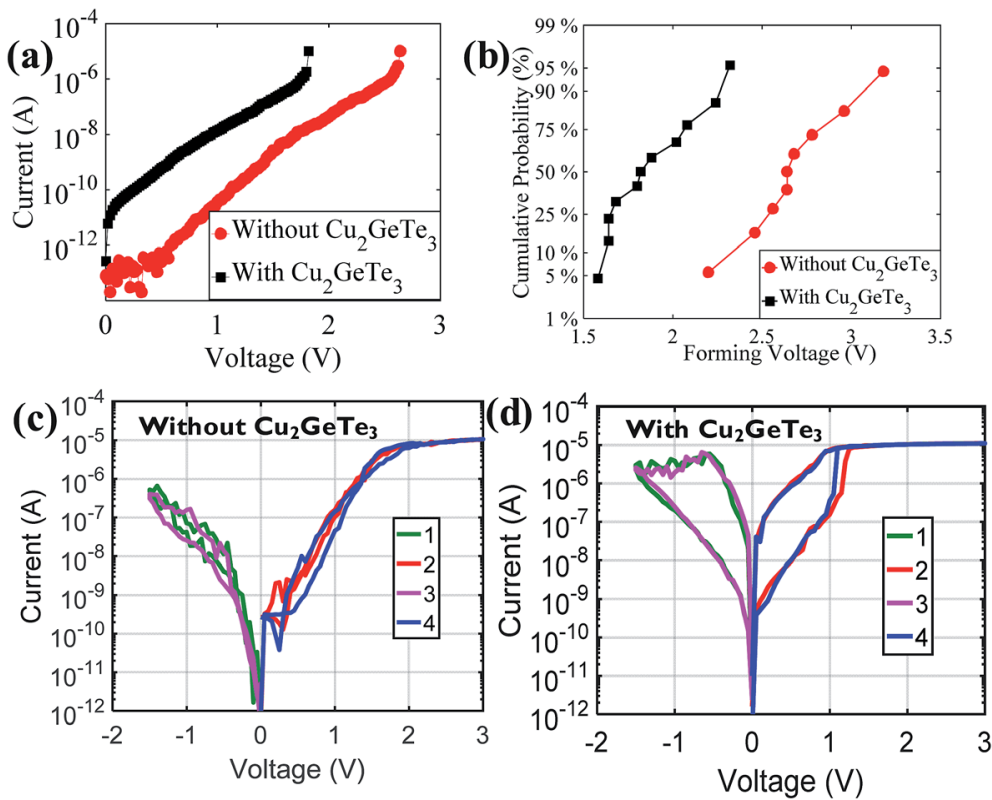

Fig. 8 Forming and switching results obtained for $\mathrm{Ge}_{50} \mathrm{Te}_{50}$ devices with or without a CGT layer; (a) median forming curves; (b) CDF $V_{f}$ plots; (c and d) DC switching characteristics obtained using a compliance current of $10 \mu \mathrm{A}$ on devices having no CGT or having a CGT Cu-supply layer, respectively.

Fig. 8 shows the results for $\mathrm{Ge}_{50} \mathrm{Te}_{50}$ devices. In summary, $V_{\mathrm{f}}$ is increased by $\sim 1 \mathrm{~V}$ for devices having no CGT and bipolar switching is suppressed, which is in agreement with results obtained for e-beam cells. Similar results were qualitatively obtained for $\mathrm{Ge}_{60} \mathrm{Se}_{40}$ devices, that is $V_{\mathrm{f}}$ is increased by $\sim 0.2 \mathrm{~V}$ (Fig. 9) and switching is suppressed for devices having no CGT.

Regarding the forming process, the initial leakage is higher in the presence of CGT for both material systems. This is explained by the in-diffusion of $\mathrm{Cu}$ into the electrolyte during the passivation processing. The presence of the CGT layer
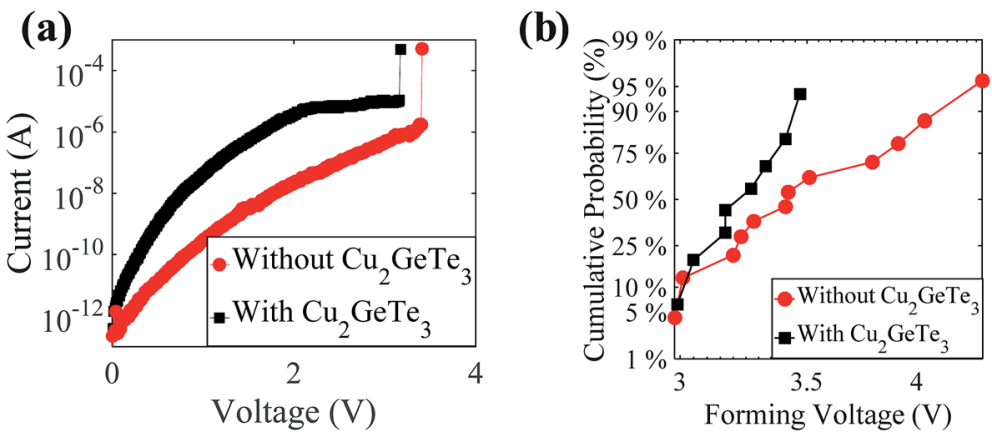

Fig. 9 Forming results obtained for $\mathrm{Ge}_{60} \mathrm{Se}_{40}$ devices with or without a CGT layer; (a) median forming curves; (b) CDF $V_{f}$ plots. 

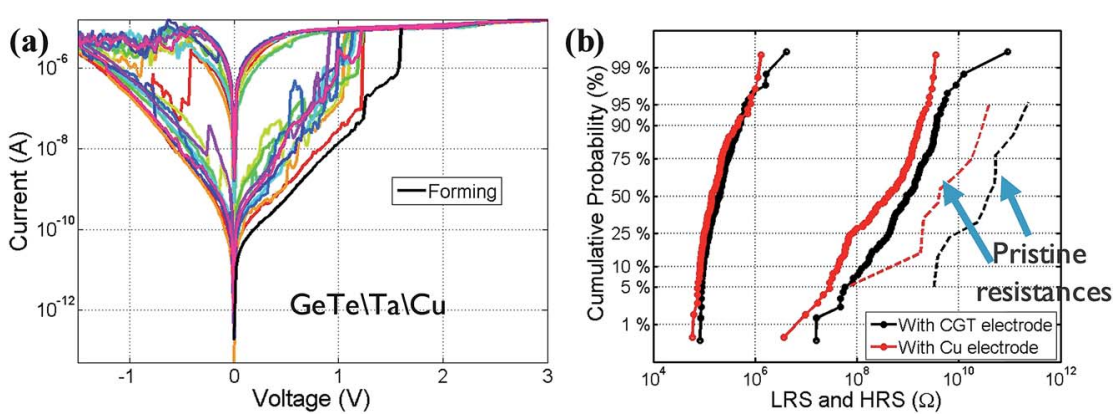

Fig. 10 (a) Switching /-V curves obtained on $\mathrm{Ge}_{50} \mathrm{Te}_{50} \backslash \mathrm{Ta}$ ICu devices, using a compliance current of $10 \mu \mathrm{A}$; (b) cumulative probability plots of HRS and LRS distributions of $\mathrm{Ge}_{50} \mathrm{Te}_{50}$ devices having either a CGT or a Cu electrode, and extracted from switching curves obtained at $10 \mu \mathrm{A}$ compliance and maximum $V_{\text {reset }}=-1.5 \mathrm{~V}$ (read voltage $=-0.1 \mathrm{~V}$ ).

substantially lowers $V_{\mathrm{f}}$ for $\mathrm{Ge}_{50} \mathrm{Te}_{50}$, however to a lesser extent for $\mathrm{Ge}_{60} \mathrm{Se}_{40}$, whereby $V_{\mathrm{f}}$ remains in the same range as without CGT, with an overlap of the distribution (Fig. 9b). As this latter material also exhibits ovonic threshold switch (OTS) properties, ${ }^{\mathbf{1 5}, 16}$ it is not excluded that the forming operation may be assisted by threshold switching, followed by $\mathrm{Cu}$ injection and $\mathrm{CF}$ formation facilitated by the joule heating induced by the current spike.

\section{Impact of the Cu-supply material on switching properties of Ge-Te devices}

Instead of CGT, some devices were integrated with sputtered Cu layers. The DC switching characteristics of $\mathrm{Ge}_{50} \mathrm{Te}_{50}$ with a pure $\mathrm{Cu}$ layer are shown in Fig. 10a. The switching characteristics are similar irrespective of the type of Cu-supply layer, as evidenced by the cumulative distributions of LRS and HRS in Fig. 10b.

Compared to the e-beam cell characteristics in Fig. 1c, the integrated $\mathrm{Ge}_{50}$ $\mathrm{Te}_{50} \backslash \mathrm{Ta} \backslash \mathrm{Cu}$ device exhibits a significantly lower RW (Fig. 6a and 10). The main structure and process differences between these devices lie in the presence of the Ta buffer layer and in the thermal budget of $250{ }^{\circ} \mathrm{C}$ applied to the integrated device. We assume that the origin of the decreased RW is related to the amount of in-diffused $\mathrm{Cu}$ (Fig. 4f), resulting in an increased pristine leakage, which limits the reset level, and which reduces the reset breakdown voltage (Fig. 6a).

\section{Write speed and retention properties}

\section{Write speed}

The time required to set the CF was evaluated for each stack. To characterize this set time, sequences of positive pulses of constant voltage amplitude $(4 \mathrm{~V})$ and logarithmically increasing pulse widths (PW) were applied to the bit line of the device in HRS state (Fig. 11a). The resistance of the device was monitored by applying a read pulse of $0.1 \mathrm{~V}$ after each programming pulse. The width of the pulse that allowed the read resistance to be decreased below $200 \mathrm{k} \Omega$ was recorded as the set time.

In both Ge-Te and Ge-Se systems, we observed an anti-correlation between the set time and the proportion of chalcogen in the electrolyte (Fig. 11b). The set time 
(a)

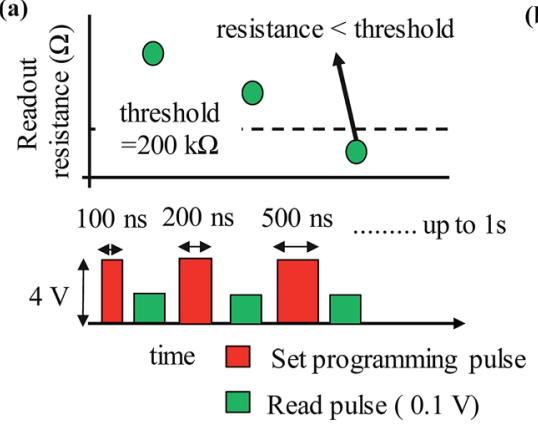

(b)

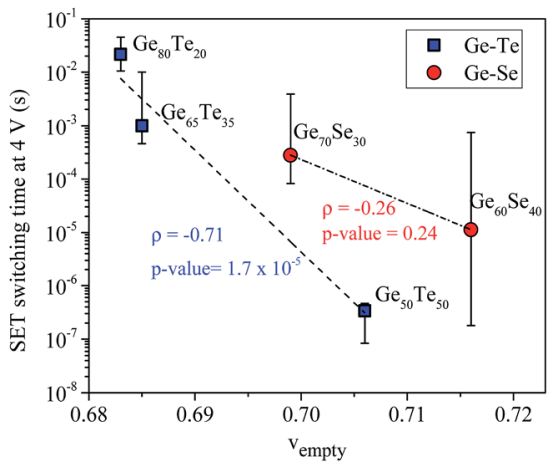

Fig. 11 (a) Device pulse programming methodology to extract the set time; (b) Correlation plot of the set time vs. the empty volume factor $\left(v_{\text {empty }}\right)$ for both $40 \mathrm{~nm}$-thick $\mathrm{Ge}-\mathrm{Te}$ and 20 nm-thick Ge-Se-based CBRAM systems.

is lower for electrolytes having a higher atomic percentage of chalcogens. Note that these set-time trends are consistent with the increased DC set voltages observed in Fig. 6 for Ge-Te and Ge-Se layers having decreasing chalcogen content.

To investigate the origin of this effect, we performed density characterizations of the chalcogenide layers (see sample schematic in Fig. 2a). This density characterization was carried out by calculation from inputs of both XRR (for thickness measurements) and high-precision mass measurements (accuracy: $10 \mu \mathrm{g}$ ) before and after layer deposition. We observed a clear decrease of the calculated layer density as a function of the chalcogen percentage. From these calculated densities we approximated the empty volume factor $\left(v_{\text {empty }}\right)$ in the amorphous electrolyte layer. This factor is defined by the ratio of unoccupied volume (dotted region in Fig. 12a) to the total volume of the electrolyte layer.

Here we would like to underline the difference between empty volume and free volume. Free volume refers to the volume in the amorphous network available for the diffusive excursion of atoms, whereas empty volume is the entire volume unoccupied by atoms. Zallen et al. ${ }^{17}$ explain this difference by considering the case of a random close packed array of atoms. The filling factor of this configuration is 0.64 , resulting in an empty volume factor of 0.36 . Here, since each atom is jam-packed, there is no volume for the atoms to move about resulting in a free volume factor $=0$. Nevertheless, there exists a monotonic relationship between the empty volume and the free volume. An increase in empty volume will increase the free volume.

The empty volume $v_{\text {empty }}$ of $\mathrm{Ge}_{a} \mathrm{X}_{b}$, where $\mathrm{X}$ is the chalcogen, is calculated from the equation shown in Fig. 12b. The volume of atoms is calculated by considering them as hard spheres with a radius equal to the covalent radius (Fig. 12c). From these calculations, we find that $v_{\text {empty }}$ is larger for electrolytes with higher chalcogen content. The summary table of density and $v_{\text {empty }}$ obtained for each electrolyte is given in Fig. 12d.

Fig. 11b shows the anti-correlation plot between the set switching time at $4 \mathrm{~V}$ and $v_{\text {empty }}$, where the strength of the correlation is quantified by Spearman's rank correlation coefficient $(\rho)$. The anti-correlation is strong $(\rho=-0.71)$ and 
(a)

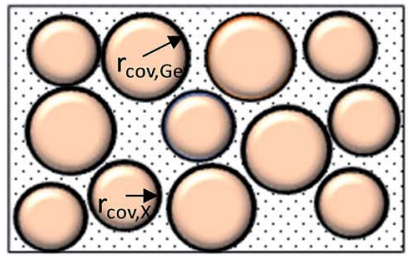

(c)

\begin{tabular}{|c|c|c|}
\hline Element & $\begin{array}{c}\mathbf{m}_{\mathbf{a}} \\
\left(\times \mathbf{1 0}^{-22} \mathbf{g}\right)\end{array}$ & $\begin{array}{c}\mathbf{r}_{\text {cov }} \\
(\tilde{\AA})\end{array}$ \\
\hline $\mathrm{Ge}$ & 1.21 & 1.22 \\
\hline $\mathrm{Te}$ & 2.12 & 1.35 \\
\hline $\mathrm{Se}$ & 1.31 & 1.16 \\
\hline
\end{tabular}

$v_{\text {empty }}=1-\frac{\text { Volume of atoms in } 1 \mathrm{~mol}}{(\text { Volume of atoms }+ \text { empty volume }) \text { in } 1 \mathrm{~mol}}$

(b)

$=1-\frac{\text { Volume of atoms in } 1 \mathrm{~mol}}{\text { Mass of atoms in } 1 \mathrm{~mol} / \text { Measured density }}$

$=1-\frac{\frac{4}{3} \pi\left(a r_{c o v, G e}^{3}+b r_{c o v, X}^{3}\right)}{\left(a m_{a, G e}+b m_{a, X}\right) / d}$

(d)

\begin{tabular}{|c|c|c|}
\hline Ele ctrolyte & $\mathbf{d}\left(\mathbf{g} / \mathbf{c m}^{3}\right)$ & $\mathbf{v}_{\text {empty }}$ \\
\hline $\mathrm{Ge}_{50} \mathrm{Te}_{50}$ & 5.529 & 0.706 \\
\hline $\mathrm{Ge}_{65} \mathrm{Te}_{35}$ & 5.616 & 0.685 \\
\hline $\mathrm{Ge}_{80} \mathrm{Te}_{20}$ & 5.403 & 0.683 \\
\hline $\mathrm{Ge}_{60} \mathrm{Se}_{40}$ & 4.940 & 0.716 \\
\hline $\mathrm{Ge}_{70} \mathrm{Se}_{30}$ & 5.108 & 0.699 \\
\hline
\end{tabular}

Fig. 12 (a) Simplified schematic of the amorphous network, with the empty volume factor corresponding to the dotted region; (b) equation used to calculate the empty volume factor; (c) atomic mass $\left(m_{\mathrm{a}}\right)$ and covalent radius $\left(r_{\text {cov }}\right)$ used in the calculation of $v_{\text {emptyi }}$ (d) summary results table of the calculated densities (from XRR and mass measurements) and calculated empty volume factor $\left(v_{\text {empty }}\right)$ of the chalcogenide layers under study.

statistically significant ( $p$-value $=0.00017$ ) for Ge-Te electrolytes. The anticorrelation is weaker $(\rho=-0.26)$ for Ge-Se electrolytes.

These results suggest that the reduction in switching time in electrolytes with a higher percentage of chalcogen is due to the increased empty volume, which would facilitate the $\mathrm{Cu}$-ion conduction. The mobility $(\mu)$ of an ion in an amorphous network is given by the expression $\mu=\gamma a^{2} \nu \exp \left(E_{\mathrm{a}} / E_{\mathrm{MN}}\right) \exp \left(-E_{\mathrm{a}} / k_{\mathrm{B}} T\right)$, where $\gamma$ is the geometric factor from random walk theory ( $=1 / 6$ in three dimensions), $a$ is the jump distance, $\nu$ is the attempt frequency, $E_{\mathrm{a}}$ is the activation energy and $E_{\mathrm{MN}}$ is the MeyerNeldel energy. ${ }^{9}$ In this equation, assuming fixed activation energy, the ion mobility increases for electrolytes with larger jump distance, i.e. the larger the empty volume in the electrolyte, the larger the cation mobility. These findings are consistent with reports showing that an increase of the free volume in the amorphous network of an ion conductor material facilitates the ion conduction. ${ }^{18}$

Another interesting observation from the correlation plot in Fig. 11b is that for a same empty volume factor, a longer time is required to set the $\mathrm{CF}$ for Ge-Se than in Ge-Te layers. In other words, Ge-Te CBRAM systems operate at lower energies. This explains also why reproducible switching in these Ge-Te devices is also obtained at operating currents as low as $10 \mu \mathrm{A}$ (Fig. 8 and 10) while no memory window is obtained for Ge-Se devices in this current range.

Hence, not only the empty volume factor but also the type of chalcogen element plays a key role in the switching speed. This will be discussed further in the next part.

\section{Retention of pulse-programmed states}

The HRS and LRS retention properties were evaluated for all electrolyte compositions investigated. Here, the programming methodology adopted was the well- 

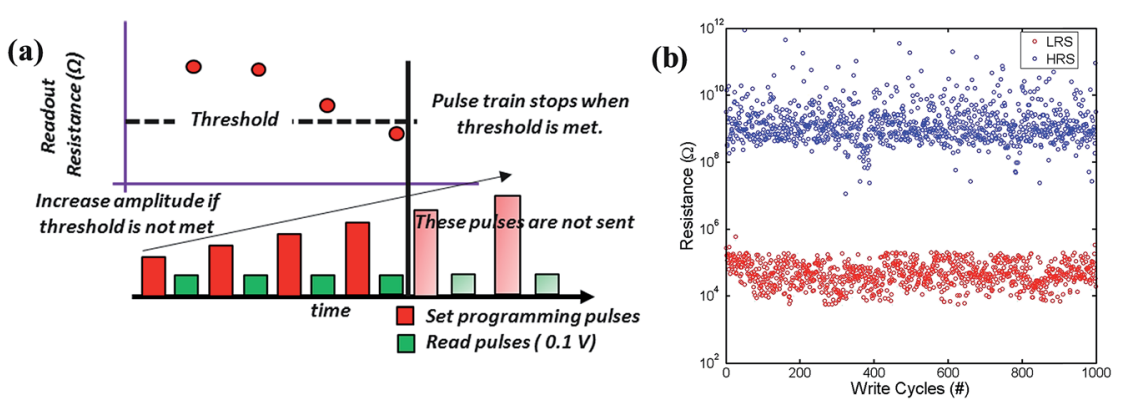

Fig. 13 (a) Increasing step pulse programming (ISPP) methodology used to program HRS and LRS states for retention assessment; (b) example of verified-programming cycling results obtained on $20 \mathrm{~nm}$-thick $\mathrm{Ge}_{60} \mathrm{Se}_{40}$ CBRAM devices using the ISPP methodology (PW $=10 \mu \mathrm{s})$.

known increasing step pulse programming (ISPP) method (Fig. 13a), consisting of applying sequences of pulses of a fixed $\mathrm{PW}=10 \mu \mathrm{s}$ but linearly incremental voltage amplitude applied to the bit line of the device (Fig. 13a). The resistance of the device was monitored by applying a read pulse of $0.1 \mathrm{~V}$ after each programming pulse. The sequence was stopped as soon as thresholds of $10 \mathrm{M} \Omega$ and 200 $\mathrm{k} \Omega$ were reached for HRS and LRS states, respectively. Fig. 13b gives an example of verified-cycling characteristics obtained on $\mathrm{Ge}_{60} \mathrm{Se}_{40}$ devices, showing effective $\mathrm{AC}$ set/reset using this ISPP methodology.

After ISPP programming, the devices were baked at $85^{\circ} \mathrm{C}$ to accelerate the state loss, and the resistance was read out at frequent time intervals. The evolution of the HRS resistance for each electrolyte composition is shown in Fig. 14 as cumulative distributions.
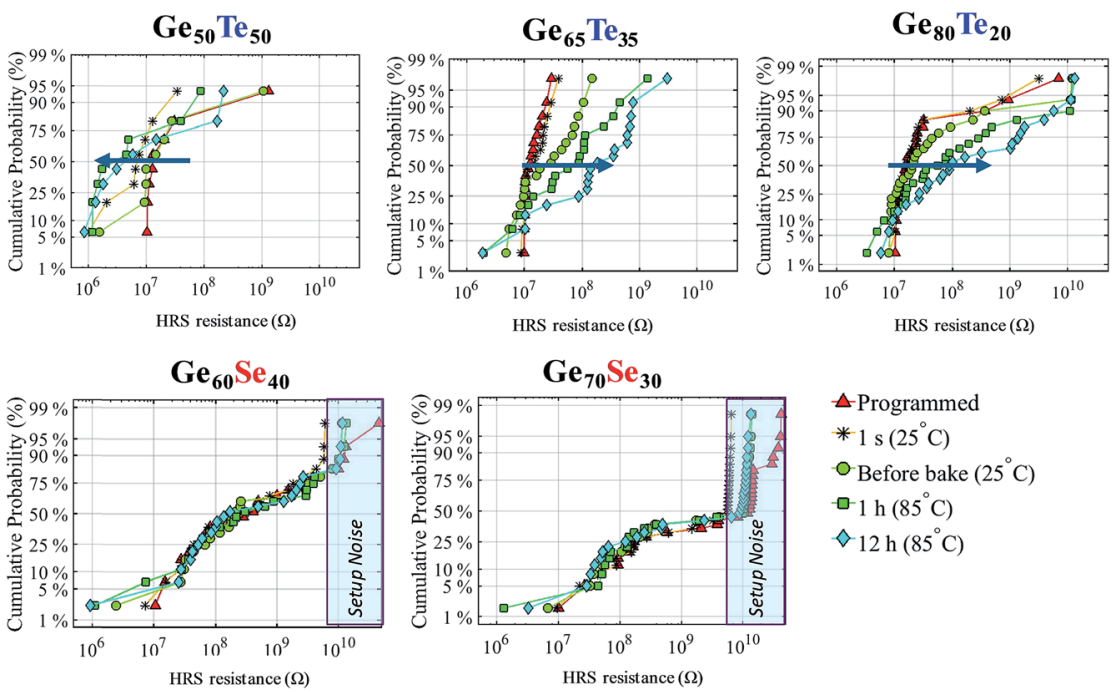

Fig. 14 Cumulative distributions of HRS states programmed using the ISPP to HRS levels $>10 \mathrm{M} \Omega$, and measured $\left(V_{\text {read }}=0.1 \mathrm{~V}\right)$ at different time intervals after baking at $85^{\circ} \mathrm{C}$; HRS drifts are observed for Ge-Te CBRAM systems while HRS stability is observed for $\mathrm{Ge}-\mathrm{Se}$ systems (note that thickness is $20 \mathrm{~nm}$ for Ge-Se devices). 


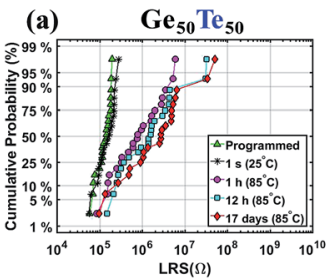

(b)
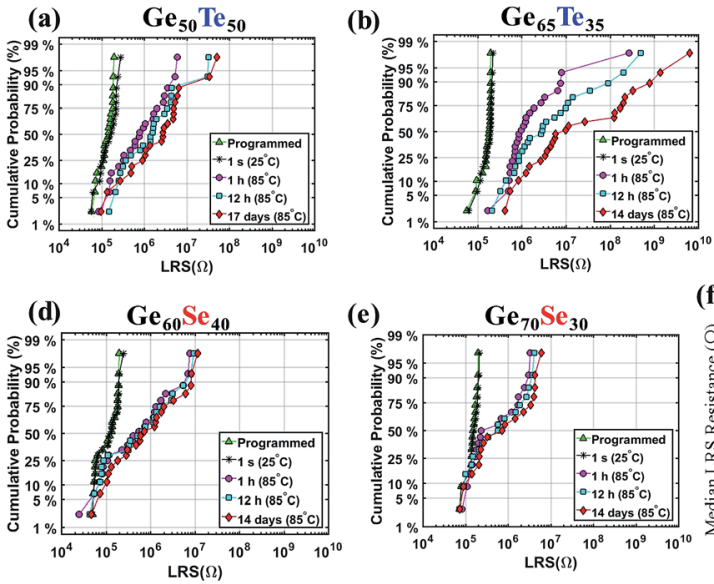

(e)

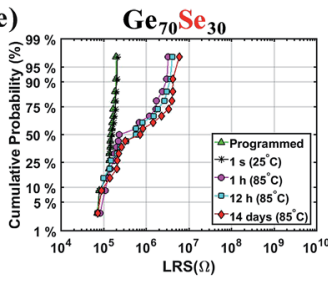

(c)

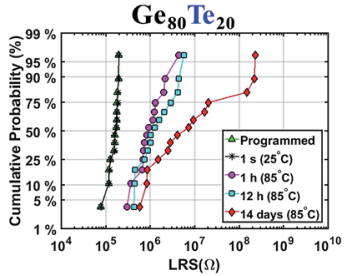

Fig. 15 (a-e) Cumulative distributions of LRS states programmed using the ISPP to LRS levels $<200 \mathrm{k} \Omega$, and measured $\left(V_{\text {read }}=0.1 \mathrm{~V}\right)$ at different time intervals after baking at $85^{\circ} \mathrm{C}$; (f) median LRS bit resistance as a function of the bake time at $85^{\circ} \mathrm{C}$ for $\mathrm{Ge}-\mathrm{Te}$ and $\mathrm{Ge}-\mathrm{Se}$ CBRAM devices (note that thickness is $20 \mathrm{~nm}$ for $\mathrm{Ge}-\mathrm{Se}$ devices).

A first observation is that the ISPP methodology confirms the improved reset efficiency observed for Ge-Se (see Fig. 7) compared to Ge-Te systems, since median levels of $>100 \mathrm{M} \Omega$ are reached for the former. Secondly, we observed that the HRS state is stable for Ge-Se electrolytes, however it drifts for Ge-Te electrolytes (Fig. 14). For $\mathrm{Ge}_{65} \mathrm{Te}_{35}$ and $\mathrm{Ge}_{80} \mathrm{Te}_{20}$ the HRS resistance increases steadily with bake time, while for $\mathrm{Ge}_{50} \mathrm{Te}_{50}$ the HRS resistance decreases suddenly on baking and shows then slow evolution with bake time.

Fig. 15a-e show the LRS counterpart retention distribution plots, and Fig. $15 f$ plots the median LRS shifts for all devices. These plots clearly show that the CF is more stable in Ge-Se than in Ge-Te devices.

To quantify the LRS stability in Ge-Se and Ge-Te devices, we evaluated the retention characteristics for $\mathrm{Ge}_{50} \mathrm{Te}_{50}$ and $\mathrm{Ge}_{60} \mathrm{Se}_{40}$ devices at three different bake temperatures $\left(85^{\circ} \mathrm{C}, 125{ }^{\circ} \mathrm{C}\right.$ and $\left.150{ }^{\circ} \mathrm{C}\right)$ in order to extract the activation energy $\left(E_{\mathrm{a}}\right)$ of the state loss.

For a better $E_{\text {a }}$ extraction accuracy, we considered the upper quartile bit, which shows more substantial drift while keeping statistical relevance (Fig. 16a). We observed that the upper quartile resistance follows a power law dependence with bake time (Fig. 16b). We defined the retention time-to-fail parameter as the time required for this resistance bit to increase by $\times 3$ from its initial ISPP-programmed value. We observed that this time-to-fail parameter follows an Arrhenius relationship (Fig. 16c and d), allowing activation energies of $E_{\mathrm{a}}=1.3 \mathrm{eV}$ for $\mathrm{Ge}_{50} \mathrm{Te}_{50}$ and $E_{\mathrm{a}}=1.9 \mathrm{eV}$ for $\mathrm{Ge}_{60} \mathrm{Se}_{40}$ devices to be extracted (Fig. 16c and d). These results suggest that $\mathrm{Cu}$ diffusivity is larger in Ge-Te layers.

To obtain more insight into this, time-of-flight secondary-ion-massspectroscopy (ToF-SIMS) depth profile experiments were carried out on Ge-X(10 $\mathrm{nm}) \backslash \mathrm{CGT}(10 \mathrm{~nm}) \backslash \mathrm{Ta}(5 \mathrm{~nm})$ stacks annealed at $400{ }^{\circ} \mathrm{C}$ for $5 \mathrm{~min}$, where $\mathrm{X}$ stands for the chalcogen element. Fig. 17a shows the resulting $\mathrm{Cu}$ signal obtained on $\mathrm{Ge}_{50} \mathrm{Te}_{50}$ and $\mathrm{Ge}_{55} \mathrm{Se}_{45}$ layers. The steeper $\mathrm{Cu}$ signal slope in $\mathrm{Ge}_{55} \mathrm{Se}_{45}$ than in $\mathrm{Ge}_{50} \mathrm{Te}_{50}$ indicates larger diffusivity of $\mathrm{Cu}$ atoms into this latter electrolyte layer. 
(a)
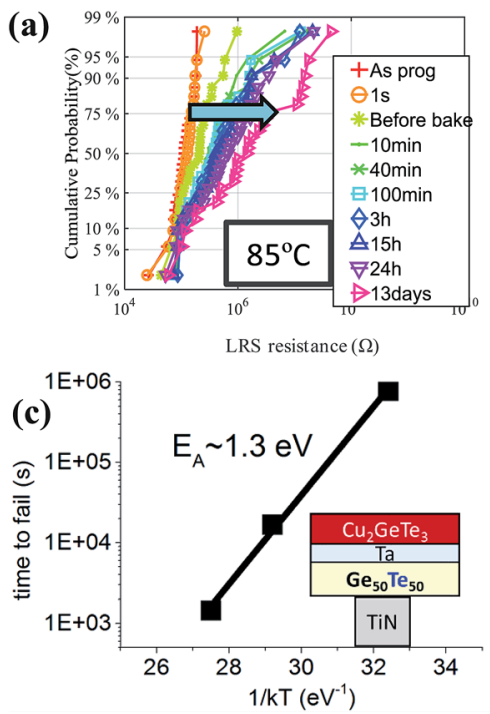

Paper
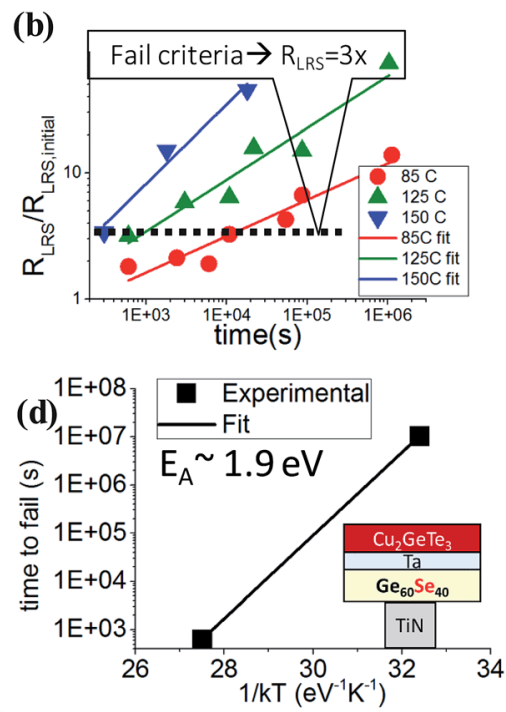

Fig. 16 Methodology used to extract the activation energy of the LRS retention loss: (a) cumulative probability plots of the LRS states after different time intervals after baking at $85^{\circ} \mathrm{C}$; (b) evolution of the upper quartile LRS bit as a function of the bake time, and extraction of the time-to-fail parameter based on the criterion of $\times 3$ increase of the bit resistance; (c) time-to-fail versus $1 / k T$ for $\mathrm{Ge}_{50} \mathrm{Te}_{50}$; (d) time-to-fail versus $1 / k T$ for $20 \mathrm{~nm}$ thick $\mathrm{Ge}_{60} \mathrm{Se}_{40}$.

This result is in agreement with the substantial amount of $\mathrm{Cu}$ evidenced by EDS in Ge-Te $\backslash$ Ta $\backslash$ CGT devices in Fig. 4f. It also supports the poor LRS retention characteristics obtained for Ge-Te devices (Fig. 15). Indeed, the $\mathrm{Cu} \mathrm{CF}$ formed in

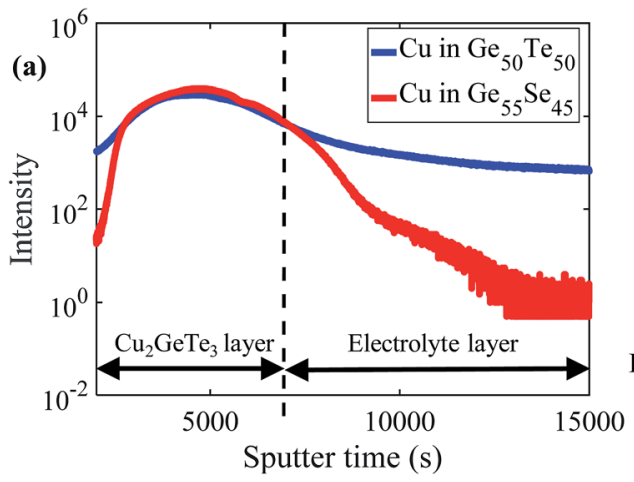

(b)

\begin{tabular}{|c|c|}
\hline Bond & $\Delta \mathbf{H}(\mathbf{k J} / \mathbf{m o l})$ \\
\hline $\mathrm{Cu}-\mathrm{Te}$ & 230.5 \\
\hline $\mathrm{Cu}-\mathrm{Se}$ & 255.2 \\
\hline $\mathrm{Cu}-\mathrm{S}$ & 274.5 \\
\hline
\end{tabular}

(c)

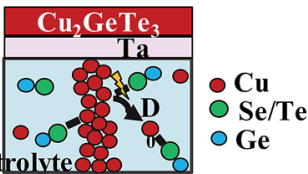

Fig. 17 (a) ToF-SIMS depth profile of $\mathrm{Cu}$ in $\mathrm{Ge}_{50} \mathrm{Te}_{50}$ and $\mathrm{Ge}_{55} \mathrm{Se}_{45}$ layers, obtained on $\mathrm{Ge}-\mathrm{X}(10 \mathrm{~nm}) \backslash \mathrm{CGT}(10 \mathrm{~nm}) \backslash \mathrm{Ta}(5 \mathrm{~nm})$ stacks annealed at $400{ }^{\circ} \mathrm{C}$ for $5 \mathrm{~min}$; the dotted line corresponds to the interface between the Cu-supply CGT layer and the chalcogenide layer; (b) bond-dissociation energy $\Delta H$ data reported from ref. 19; (c) schematic of the hypothesized diffusion mechanism of $\mathrm{Cu}$ from the CF surface outwards through the electrolyte layer, by means of successive $\mathrm{Cu}$-chalcogen bond-breaking and bondformation. 
the electrolyte layer in the LRS state will more easily diffuse sideways for Ge-Te than for Ge-Se layers. This out-diffusion mechanism likely explains, as well, the HRS retention drift towards higher resistance levels observed for Ge-rich Ge-Te devices (Fig. 14). The origin of the HRS drift towards lower resistances observed for $\mathrm{Ge}_{50} \mathrm{Te}_{50}$ is, however, not clear and requires further investigation.

\section{Discussion and guidelines for device improvements}

Clearly, from this study Ge-Se layers prove more attractive than Ge-Te layers for CBRAM application, due to the larger memory window and better retention properties. These promising properties are of course traded by large switching energy, however this may be further improved using thinner $(<20 \mathrm{~nm})$ layers and by exploring Se-rich Ge-Se electrolytes, which should increase further the empty volume factor and thus decrease the set time (Fig. 11b). An additional benefit is the further improved thermal stability of Se-rich Ge-Se layers, reported in ref. 16, which would thus facilitate further the integration flow.

As for Ge-Te devices, their properties may be improved by further tuning of the Cu-buffer layer material and thickness, in order to better limit the amount of indiffused $\mathrm{Cu}$ material after integration. This might directly benefit the reset efficiency, the memory window as well as the HRS breakdown robustness.

Regarding the physics at the origin of the device properties, interestingly the bond-dissociation energy $(\Delta H)$ reported for $\mathrm{Cu}-\mathrm{Se}$ is larger than for $\mathrm{Cu}-\mathrm{Te}$ (table in Fig. 17b). The stronger interaction between $\mathrm{Cu}$ and Se may increase the activation barrier of $\mathrm{Cu}$ movement $\left(E_{\mathrm{a}}\right)$ through the network, thereby reducing its mobility and increasing the set energy (time and voltage). This also suggests that a $\mathrm{Cu}$ filament formed in the Ge-Se matrix is stabilized by strong bond formation between the $\mathrm{Cu}$ filament surface and the neighboring Se elements in the electrolyte layer, resulting in higher activation energy $\left(E_{\mathrm{a}}\right)$ of the LRS retention loss.

From these considerations, the type of chalcogen used in the electrolyte layer and its bonding strength with $\mathrm{Cu}$ will play key roles in both retention and switching energy properties. Fig. 17c shows a possible mechanism of $\mathrm{Cu}$ motion from the $\mathrm{CF}$ surface, which is driven by successive $\mathrm{X}-\mathrm{Cu}$ bond breaking and formation ( $\mathrm{X}=\mathrm{Se}$ or $\mathrm{Te})$ and which would control both the retention properties and the $\mathrm{Cu}$ cation motion during set.

Following this supposed mechanism, Ge-S electrolyte systems are possible candidates to allow the retention properties to be further improved, thanks to the larger bond dissociation energy for $\mathrm{Cu}-\mathrm{S}$ than for $\mathrm{Cu}-\mathrm{Se}$ (Fig. 17b). For example, Ge-S has been developed in combination with $\mathrm{Ag}$ active species for highperformance CBRAM. ${ }^{20}$

Finally, it is important to mention as a last note that most recent technology advances tend to indicate that oxide electrolyte solutions seem to outperform chalcogenide electrolytes developed so far. ${ }^{21,22}$

\section{Conclusions}

In this study we showed that the chalcogen element plays a critical role in determining the retention and switching energy of the CBRAM device. Firstly, we showed that the type of chalcogen element impacts the time required for filament formation (set time) and filament stability (LRS retention). Ge-Se electrolytes 
exhibit longer set times however better LRS retention than Ge-Te electrolytes, which we attributed to stronger $\mathrm{Cu}-\mathrm{Se}$ bonds compared to $\mathrm{Cu}-\mathrm{Te}$ bonds. Secondly, for a fixed chalcogenide system, we showed an anti-correlation relationship between the set time and the proportion of chalcogen in the electrolyte. We demonstrated this effect by the decreased material density induced by larger chalcogenide percentage in the layer, resulting in a faster $\mathrm{Cu}$ cation motion during set switching.

\section{Conflicts of interest}

There are no conflicts to declare.

\section{References}

1 M. Kund, G. Beitel, C. U. Pinnow, T. Rohr, J. Schumann, R. Symanczyk, K. Ufert, and G. Muller, In Electron Devices Meeting (IEDM), 2005, pp. 754-757.

2 K. Aratani, K. Ohba, T. Mizuguchi, S. Yasuda, T. Shiimoto, T. Tsushima, T. Sone, K. Endo, A. Kouchiyama, S. Sasaki and A. Maesaka, In Electron Devices Meeting (IEDM), 2007, pp. 783-786.

3 R. Symanczyk, R. Bruchhaus, R. Dittrich and M. Kund, IEEE Electron Device Lett., 2009, 30(8), 876.

4 A. Belmonte, A. Fantini, A. Redolfi, M. Houssa, M. Jurczak and L. Goux, Phys. Status Solidi A, 2016, 213(2), 302-305.

5 M. N. Kozicki, M. Park and M. Mitkova, IEEE Trans. Nanotechnol., 2005, 4(3), 331-338.

6 S. Z. Rahaman, S. Maikap, H. C. Chiu, C. H. Lin, T. Y. Wu, Y. S. Chen, P. J. Tzeng, F. Chen, M. J. Kao, and M. J. Tsai, In Memory Workshop (IMW), 2009, pp. 1-4.

7 D. Kamalanathan, U. Russo, D. Ielmini and M. N. Kozicki, IEEE Electron Device Lett., 2009, 30(5), 553-555.

8 S. J. Choi, J. H. Lee, H. J. Bae, W. Y. Yang, T. W. Kim and K. H. Kim, IEEE Electron Device Lett., 2009, 30(2), 120-122.

9 K. Tanaka and K. Shimakawa, Amorphous chalcogenide semiconductors and related materials, Springer Science \& Business Media, 2011.

10 W. Devulder, K. Opsomer, G. Rampelberg, B. De Schutter, K. Devloo-Casier, M. Jurczak, L. Goux and C. Detavernier, J. Mater. Chem. C, 2015, 3(48), 12469-12476.

11 M. H. R. Lankhorst, J. Non-Cryst. Solids, 2002, 297(2-3), 210-219.

12 M. Vlček, L. Tichý, J. Klikorka and A. Tř́ska, J. Mater. Sci. Lett., 1988, 7(4), 335338.

13 A. Fantini, V. Sousa, L. Perniola, E. Gourvest, J. C. Bastien, S. Maitrejean, et al., In Electron Devices Meeting (IEDM), 2010, pp. 644-647.

14 A. Belmonte, W. Kim, B. T. Chan, N. Heylen, A. Fantini, M. Houssa, M. Jurczak and L. Goux, IEEE Trans. Electron Devices, 2013, 60(11), 3690-3695.

15 S. Clima, B. Govoreanu, K. Opsomer, A. Velea, N. S. Avasarala, W. Devulder, I. Shlyakhov, G. L. Donadio, T. Witters, S. Kundu and L. Goux, In Electron Devices Meeting (IEDM), 2017, pp. 79-82.

16 N. S. Avasarala, G. L. Donadio, T. Witters, K. Opsomer, B. Govoreanu, A. Fantini, et al., Symposium on VLSI Technology Digest of Technical Papers, 2018. 
17 R. Zallen, The physics of amorphous solids, John Wiley \& Sons, 2008, pp. 212218.

18 J. Swenson and L. Börjesson, Phys. Rev. Lett., 1996, 77(17), 3569.

19 Huheey and B. d. Darwent, National Standard Reference Data Series, The Strengths of Chemical Bonds, National Bureau of Standards, no. 31, Washington, DC, 1958, pp. A-21 to A-34; S. W. Benson, J. Chem. Educ., 1965, 42, 502 .

20 C. Gopalan, Y. Ma, T. Gallo, J. Wang, E. Runnion, J. Saenz, F. Koushan, P. Blanchard and S. Hollmer, Solid-State Electron., 2011, 58, 54-61.

21 J. R. Jameson, P. Blanchard, C. Cheng, J. Dinh, A. Gallo, V. Gopalakrishnan, C. Gopalan, B. Guichet, S. Hsu, D. Kamalanathan, D. Kim, et al., In Electron Devices Meeting (IEDM), 2013.

22 S. Sills, S. Yasuda, A. Calderoni, C. Cardon, J. Strand, K. Aratani and N. Ramaswamy, Symposium on VLSI Technology Digest of Technical Papers, 2015. 